Agron. Mesoam. 29(1):63-74. Enero-abril, 2018

ISSN 2215-3608, doi:10.15517/ma.v29i1.28205

http://www.revistas.ucr.ac.cr/index.php/agromeso

\title{
Diversidad morfológica de semilla y fruto de diez colectas mexicanas de Lagenaria siceraria ${ }^{1}$
}

\section{Seed and fruit morphological diversity of ten Mexican Lagenaria siceraria landraces}

\author{
Onécimo Grimaldo-Juárez z, Ángel Manuel Suárez-Hernández², Carlos Ceceña-Durán², \\ Daniel González-Mendoza ${ }^{2}$
}

\begin{abstract}
Resumen
Lagenaria siceraria presenta gran diversidad morfológica de semilla y fruto, por lo que, es necesario agruparla en morfotipos de acuerdo con la similitud en caracteres. El objetivo del estudio fue analizar y calificar la variación morfológica de semilla y fruto de diez colectas de Lagenaria siceraria, originarias de diferentes regiones de México. La investigación se realizó en el campo experimental del Instituto de Ciencias Agrícolas de la Universidad Autónoma de Baja California, México, durante el ciclo primavera-verano del 2014. Se evaluaron diecisiete caracteres cuantitativos de fruto y semilla, analizados con técnicas multivariantes. La variación fenotípica de $L$. siceraria se identificó principalmente por la longitud y diámetro de semilla, y en fruto por la longitud y circunferencia inferior, se formaron dos grupos a niveles de disimilitud de 0,76 y 0,69, respectivamente. El primero se integró por las colectas L45, L46, L47 y L56, que presentaron menor dimensión en fruto y semilla, con alto nivel de similitud entre los materiales. El segundo se formó con las colectas L43, L48, L50, L53, L54 y L55, el cual fue más variable y presentó mayores dimensiones que el anterior. Lo anterior muestra que hay variabilidad genética en los materiales nativos de México.
\end{abstract}

Palabras clave: divergencia genética, Cucurbitaceae, frutas cucurbitáceas, características de semillas.

\begin{abstract}
Lagenaria siceraria presents a great morphological diversity of seed and fruit, so it is necessary to group them in morphotypes according to the similarities in characters. The objective of this study was to analyze and quantify the morphological variation of seed and fruit of ten collections of L. siceraria, from different Mexican regions. The research was conducted in the experimental field of the Agricultural Sciences Institute of the Autonomous University Baja California, Mexico, during the spring-summer 2014 cycle. Seventeen quantitative fruit and seed characters were evaluated, and analyzed with multivariate techniques. The phenotypic variation of L. siceraria was identified mainly by the length and diameter of seed, and in fruit by the length and lower circumference. Two groups were formed at levels of dissimilarity of 0,76 and 0,69, respectively. The first group was integrated by collections L45, L46, L47, and L56, which had smaller dimensions in fruit and seed, with a higher level of similarity between the materials. The second group was formed by L43, L48, L50, L53, L54, and L55 landraces, this group had more variables and
\end{abstract}

1 Recibido: 6 de octubre, 2016. Aceptado: 17 de marzo, 2017. Este trabajo formó parte de la tesis doctoral en Ciencias Agropecuarias del segundo autor. Universidad Autónoma de Baja California. Mexicali, Baja California, México

2 Universidad Autónoma de Baja California, Instituto de Ciencias Agrícolas. Baja California, México, C.P 21705. onecimo.grimaldo@uabc. edu.mx,manuel.suarez@uabc.edu.mx,carlos.cecena.duran@uabc.edu.mx, danielg@uabc.edu.mx. 
presented larger dimensions than previous one. The aforementioned shows that there exists a genetic variability in the Mexico's native L. siceraria materials.

Keywords: genetic divergence, Cucurbitaceae, cucurbit fruits, seed characteristics.

\section{Introducción}

Lagenaria siceraria es una cucurbitácea conocida regionalmente en México como guaje, bule o tecomate. La importancia de esta especie son sus propiedades medicinales y el uso de los frutos como recipiente y artesanía decorada (Teppner, 2004; Guzmán-Hernández et al., 2016). El fruto desecado es abierto para extracción de la semilla y restos de pulpa, después es usado como recipiente para el almacenamiento de agua y granos (Bevacqua, 1994). También es empleado como instrumento musical en eventos religiosos y artesanalmente es decorado y comercializado en mercados regionales (Chimonyo y Modi, 2013).

En África, Asia, y América, diversas investigaciones coinciden en señalar que Lagenaria es considerada una de las primeras especies cultivadas por el hombre. Estudios arqueológicos evidencian que, su cultivo en África se estima entre los años 4000 a 5000 AC, en Asia 6000 a 10000 AC y en América 8000 a 10000 AC (Decker-Walters et al., 2001; Erickson et al., 2005; Simth, 2005). La distribución de esta especie en el continente americano se considera que fue producto de la dispersión natural a través de las corrientes marítimas y oceánicas (Kistler et al., 2014). Estudios moleculares demuestran que germoplasmas americanos de Lagenaria tienen orígenes mezclados de materiales africanos y asiáticos (Gürcan et al., 2015). El género Lagenaria integra seis especies, de las cuales L. siceraria es la más variable, principalmente en fruto y semilla. La forma del fruto varía de esférica a alargada, oblonga, forma dos cuerpos divididos por una constricción (Decker-Walters et al., 2001; Morimoto et al., 2005). Las semillas difieren en tamaño, peso y color del margen (Yetisir et al., 2008).

El guaje (L. siceraria) es considerada como la única especie cultivada en México desde tiempos precolombinos, no nativa de América. Esta planta ha sido ampliamente utilizada como alimento, debido a la presencia de fitoquímicos con propiedades hepatoprotectivas, cardioprotectoras, entre otras que la hacen una planta de gran interés etnofarmacológico (Kubde et al., 2010; Guzmán-Hernández et al., 2016). Por otra parte, estudios recientes muestran el potencial agrícola de esta planta al ser empleada como portainjerto en la producción de sandía (Citrullus lanatus); debido a la tolerancia que presenta L. siceraria al establecimiento de hongos, como la pudrición causada por diferentes especies de Fusarium (Miguel et al., 2004; Yetisir et al., 2007); así como la inducción de mejoras en parámetros de rendimiento y calidad de la fruta en donde se emplea como portainjerto (Yetisir y Sari, 2003; Karaca et al., 2012).

En México el estudio de L. siceraria como portainjerto en plantas de interés agrícola es relativamente nuevo, se ha enfocado principalmente en evaluar su efecto en parámetros físico-químicos en los frutos de diferentes variedades de sandía o diferentes densidades de siembra (López-Elías et al., 2011; Suárez-Hernández et al., 2017). Sin embargo, son escasos estudios que han analizado la diversidad morfológica de materiales nativos de L. siceraria de México. Por lo anterior, el objetivo del presente estudio fue analizar y clasificar la variación morfológica de semilla y fruto de diez colectas de L. siceraria, originarias de diferentes regiones de México. 


\section{Materiales y métodos}

Las colectas de frutos de L. siceraria se realizaron en los estados de Oaxaca, San Luis Potosí, Morelos y Baja California (Cuadro 1), las cuales consistieron de dos a cuatro frutos a los que se les extrajo la semilla. En el Campo Experimental del Instituto de Ciencias Agrícolas de la Universidad Autónoma de Baja California, se sembraron cincuenta semillas por colecta en el periodo primavera-verano del año 2014. La siembra se realizó en charolas germinadoras de unicel de $67,5 \mathrm{~cm}$ de largo, $33,5 \mathrm{~cm}$ de ancho y 7,3 cm de alto. Cada charola tenía 128 cavidades de forma cónica con dimensiones de $3,8 \mathrm{~cm}$ x $3,8 \mathrm{~cm}$ y capacidad de $55 \mathrm{ml}$. Se seleccionaron veinticinco plántulas en la etapa de dos hojas verdaderas, con uniformidad en altura y sanidad, de un total de cincuenta plántulas establecidas por colecta. Las plántulas fueron establecidas a su lugar definitivo en campo, donde la distribución fue en hileras separadas a $150 \mathrm{~cm}$ y una distancia entre plantas de $50 \mathrm{~cm}$.

Cuadro 1. Clave y procedencia de colectas mexicanas de L. siceraria, empleadas en un estudio de diversidad morfológica. Campo Experimental del Instituto de Ciencias Agrícolas, Universidad Autónoma de Baja California, México. 2014.

Table 1. Key and origin of Mexican L. siceraria landraces used on this morphological diversity study. Agricultural Sciences Institute Experimental field, Autonomous University of Baja California, Mexico. 2014.

\begin{tabular}{ll}
\hline Clave & Procedencia \\
\hline L43 & Poblado Díaz Ordaz, Ensenada, Baja California \\
L45 & Mexicali, Baja California \\
L46 & Silacayoapan, Oaxaca \\
L47 & Mexicali, Baja California \\
L48 & Camalú Baja California \\
L50 & Camalú Baja California \\
L53 & Ejido Nuevo León - Mexicali, Baja California \\
L54 & Río Verde, San Luis Potosí \\
L55 & Ejido Nuevo León - Mexicali, Baja California \\
L56 & Cuautla, Morelos \\
\hline
\end{tabular}

El manejo del cultivo fue en espalderas, establecidas con barrotes de madera y rafia. El suministro de agua y nutrientes se realizó mediante el uso de cinta de riego, con goteros a $30 \mathrm{~cm}$ de separación y un caudal de emisor de un LPH. Los riegos se aplicaron continuamente, con base en la tensión matricial del suelo, determinada con tensiómetros a profundidades de 30 y $45 \mathrm{~cm}$, se consideró como límite inferior de tensión de humedad a $10 \mathrm{kPa}$ (inicio del riego) y límite superior a $5 \mathrm{kPa}$ (final del riego). La fertilización se realizó con la fórmula comercial 80:40:60, de acuerdo con las recomendaciones de Kelley et al. (2009) para el cultivo de calabaza. En cuanto a plagas y enfermedades, no se registraron incidencias durante el ciclo biológico del cultivo. El control de malezas fue manual.

En la fase de floración de las plantas y, en específico, en el desarrollo de botón floral, se cubrieron botones masculinos y femeninos en cada una de las plantas, posteriormente, en antesis las flores se polinizaron manualmente con polen proveniente de plantas de la misma colecta. En cada planta se mantuvieron de tres a cinco frutos hasta su madurez; después, los frutos cosechados fueron sometidos a un periodo de secado para la extracción de semillas. En cada colecta se tomaron al azar veinte frutos y cien semillas, cuantificándose los caracteres indicados en el Cuadro 2. 
Cuadro 2. Caracteres evaluados en frutos y semillas de L. siceraria, empleados en un estudio de diversidad morfológica. Campo Experimental del Instituto de Ciencias Agrícolas, Universidad Autónoma de Baja California, México. 2014.

Table 2. Evaluated characters in fruits and seeds of L. siceraria, used on this morphological diversity. Agricultural Sciences Institute Experimental field, Autonomous University of Baja California, Mexico. 2014.

\begin{tabular}{clc}
\hline Núm. & Carácter & Abreviación \\
\hline 1 & Longitud de pedúnculo de fruto $(\mathrm{cm})$ & LPED \\
2 & Longitud de fruto $(\mathrm{cm})$ & LFR \\
3 & Circunferencia superior de fruto $(\mathrm{cm})$ & CSUP \\
4 & Circunferencia inferior de fruto $(\mathrm{cm})$ & CINF \\
5 & Circunferencia de cintura de fruto $(\mathrm{cm})$ & CTU \\
6 & Volumen de fruto $(\mathrm{ml})$ & VF \\
7 & Número de semillas por fruto & NSF \\
8 & Peso de cien semillas $(\mathrm{g})$ & PCS \\
9 & Peso de testa de semilla $(\mathrm{g})$ & PTS \\
10 & Peso de almendra de semilla $(\mathrm{g})$ & PAS \\
11 & Longitud de semilla $(\mathrm{cm})$ & LS \\
12 & Ancho de semilla $(\mathrm{cm})$ & AS \\
13 & Grosor de semilla $(\mathrm{cm})$ & GS \\
14 & Esfericidad de semilla & ESS \\
15 & Diámetro aritmético semilla $(\mathrm{cm})$ & DAS \\
16 & Relación AS/LS de semilla $(\%)$ & RALS \\
17 & Área estimada de semilla $\left(\mathrm{cm}^{2}\right)$ & AES \\
\hline
\end{tabular}

En las variables de fruto, la circunferencia superior e inferior se refiere a las dos partes separadas por la constricción del fruto, tal como se aprecia en la Figura 1.

Variables de semilla como esfericidad, diámetro aritmético, relación largo ancho y área, se cuantificaron mediante las ecuaciones propuestas por Pradhan et al. (2013).

a) Esfericidad de semilla (ESS) expresado en porcentaje.

$$
E S S=\frac{(L S \times A S \times G S)^{1 / 3}}{L S}
$$

b) Diámetro aritmético de la semilla (DAS).

$$
D A S=\frac{L S+A S+G S}{3}
$$

c) Relación ancho/largo de semilla (RALS).

$$
R A L S=A S / L S \times 100
$$




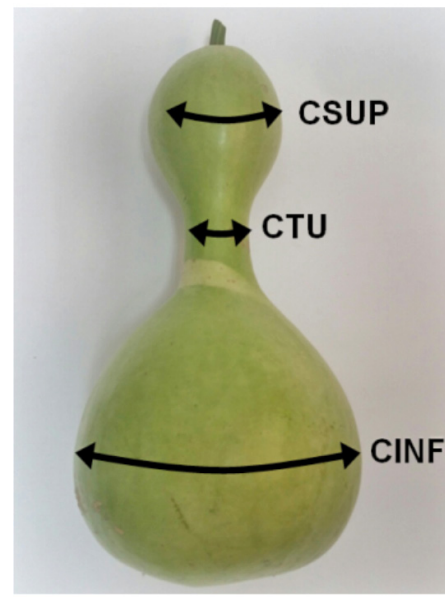

Figura 1. Caracteres de circunferencia superior (CSUP), de cintura (CTU) e inferior (CINF) evaluados en fruto de L. siceraria. Campo Experimental del Instituto de Ciencias Agrícolas, Universidad Autónoma de Baja California, México. 2014.

Figure 1. Evaluated characteristics of upper circumference (CSUP), waist (CTU), and inferior circumference (CINF) in L. siceraria fruit. Agricultural Sciences Institute Experimental field, Autonomous University of Baja California, Mexico. 2014.

d) Área de la semilla (AES).

$$
A E S=\pi D_{g}^{2}
$$

Donde, el diámetro geométrico (Dg) se estima como:

$$
D g=(L S \times A S \times G S)^{1 / 3}
$$

La información generada fue analizada mediante análisis de componentes principales (ACP), con el propósito de identificar las variables de mayor importancia en la variación de las colectas de L. siceraria. Además, se realizó un análisis discriminante canónico y un análisis de agrupamiento, mediante la técnica de UPGMA. Todos los análisis se realizaron con el paquete estadístico SAS v. 9.2 (SAS, 2009).

\section{Resultados}

En la variación morfológica de las diez colectas analizadas mediante caracteres de fruto y semilla (Cuadro 3), se encontró que los dos primeros componentes explicaron el $82 \%$ de la variación (CP1=72,21\% y CP2=9,81\%). En el primer componente se identificó en fruto a los caracteres volumen y circunferencia de la parte superior e inferior como los de mayor contribución a la variación, y en el caso de la semilla, el peso de 100 semillas, longitud, diámetro y área estimada. El segundo componente únicamente se asoció con la longitud de fruto. En la dispersión gráfica de las colectas (Figura 2), se observa que en el componente 1, los materiales L43, L48, L50, L53, L54 y 
Cuadro 3. Vectores propios de los dos primeros componentes principales (CP), en el análisis de diecisiete caracteres de fruto y semilla evaluados en diez colectas mexicanas de L. siceraria. Campo Experimental del Instituto de Ciencias Agrícolas, Universidad Autónoma de Baja California, México. 2014.

Table 3. Vectors of the first two principal components (PC), in the analysis of seventeen characters of fruit and seed evaluated in ten Mexican L. siceraria landraces. Agricultural Sciences Institute Experimental field, Autonomous University of Baja California, Mexico. 2014.

\begin{tabular}{lcc}
\hline Carácter & CP 1 & CP 2 \\
\hline Longitud de pedúnculo (LPED) & 0,21 & $-0,37$ \\
Longitud de fruto (LFR) & 0,17 & 0,55 \\
Circunferencia superior de fruto (CSUP) & 0,26 & $-0,12$ \\
Circunferencia inferior de fruto (CINF) & 0,26 & 0,17 \\
Circunferencia de cintura de fruto (CTU) & 0,23 & $-0,27$ \\
Volumen de fruto (VF) & 0,26 & 0,04 \\
Numero de semillas por fruto (NSF) & 0,20 & 0,37 \\
Peso de 100 semillas (PCS) & 0,25 & 0,19 \\
Peso de testa de semilla (PTS) & 0,22 & $-0,04$ \\
Peso de almendra de semilla (PAS) & 0,23 & 0,03 \\
Longitud de semilla (LS) & 0,27 & $-0,10$ \\
Ancho de semilla (AS) & 0,21 & 0,30 \\
Grosor de semilla (GS) & 0,19 & $-0,19$ \\
Esfericidad de semilla (ESS) & $-0,23$ & 0,13 \\
Diámetro aritmético de semilla (DAS) & 0,28 & $-0,02$ \\
Relación ancho/largo de semilla (RALS) & 0,18 & $-0,33$ \\
Área estimada de semilla (AES) & 0,27 & $-0,01$ \\
\hline
\end{tabular}

L55, comparten características similares, ya que, presentaron semillas grandes de longitudes de 1,78 a 2,22 cm, diámetro aritmético de 1,00 a 1,15 cm, área estimada de 2,08 a 2,61 $\mathrm{cm}^{2}$ y peso de 100 semillas de 18,01 a $24,93 \mathrm{~g}$. Estas semillas provenían de frutos de mayor volumen que registraron de 1981,75 a 3353,75 ml y circunferencia de la parte superior del fruto de 27,65 a 36,18 cm e inferior de 60,95 a 71,63 cm, con respecto al grupo formado por las colectas L45, L46, L47 y L56, que se caracterizaron por contener frutos y semillas de menores dimensiones. La longitud de semillas fue de 1,17 a $1,48 \mathrm{~cm}$, diámetro aritmético de 0,70 a $0,84 \mathrm{~cm}$ y área de 1,15 a $1,67 \mathrm{~cm}^{2}$, en frutos el volumen varió de 309,33 a $1185,71 \mathrm{ml}$, circunferencia superior de 17,37 a 23,91 cm y circunferencia inferior de 29,86 a 48,03 cm. En el componente 2, solo la colecta L53, se diferenció de las demás por presentar mayor longitud de fruto de 39,97 a 49,53 cm, ya que, el resto de las colectas presentó $34 \%$ menos longitud.

En el análisis discriminante canónico de las diez colectas (Cuadro 4), se ratificó que la circunferencia inferior del fruto, longitud y diámetro aritmético de semilla, fueron los de mayor importancia en la variación de L. siceraria, de acuerdo con la primera función discriminante canónica que registró el 77\% de la variación total. En lo que respecta a la función discriminante 2, la contribución en la variación fue del $10 \%$, atribuyéndose a diferencias en la longitud del fruto entre las colectas. La similitud en las características de las colectas identificadas por el análisis discriminante canónico, formó grupos de 2 y 3 materiales (Figura 3 y Cuadro 5). Uno de los grupos lo integró las colectas L43, L50 y L54, las cuales presentaron valores de longitud de fruto de 26,79 a 29,01 cm, circunferencia inferior de 60,95 a 71,63 cm, longitud de semilla de 2,07 a 2,15 cm y diámetro aritmético de 1,06 a 1,15 cm. Un segundo grupo se formó con las colectas L45 y L47, que tuvieron menores dimensiones que el grupo anterior, por 


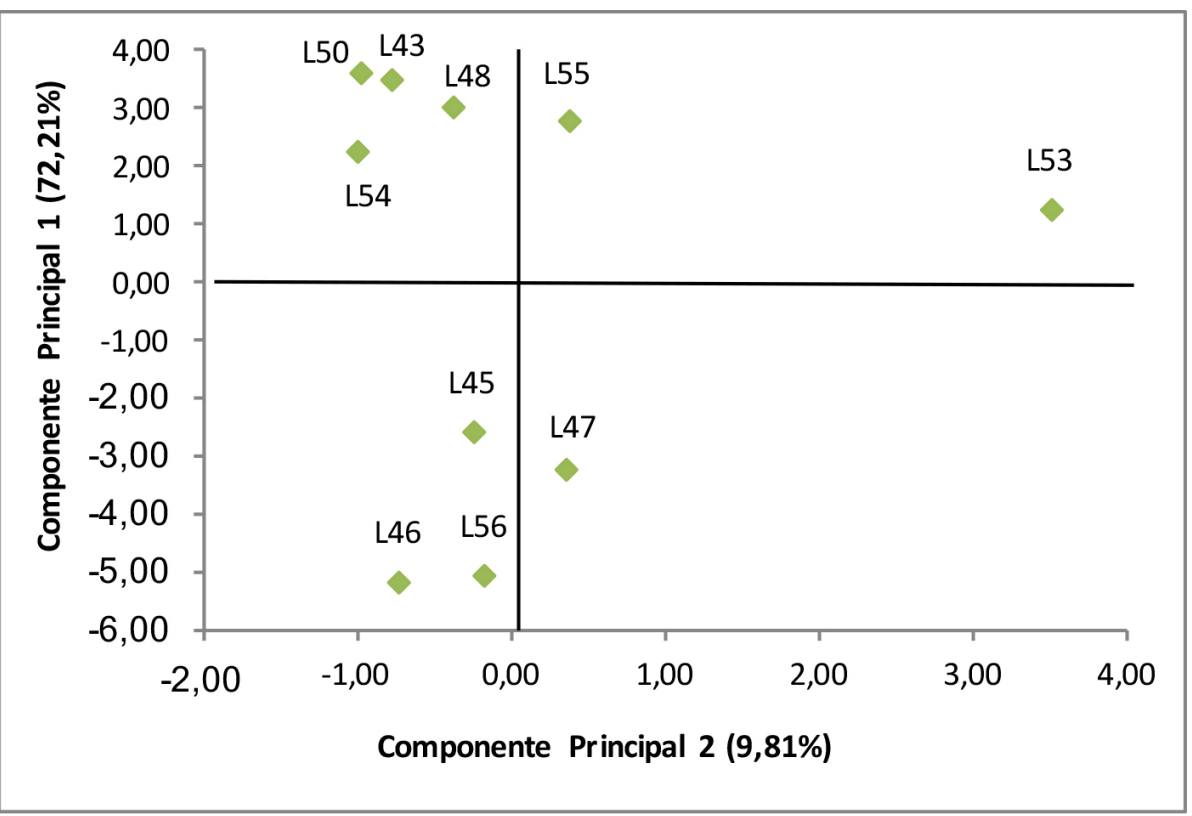

Figura 2. Distribución de diez colectas mexicanas de L. siceraria, con base al análisis de diecisiete caracteres en los dos primeros componentes principales. Campo Experimental del Instituto de Ciencias Agrícolas, Universidad Autónoma de Baja California, México. 2014.

Figure 2. Distribution of ten Mexican L. siceraria landraces based on the analysis of seventeen characters regarding their first two principal components. Agricultural Sciences Institute Experimental field, Autonomous University of Baja California, Mexico. 2014.

Cuadro 4. Coeficientes canónicos estandarizados de las dos primeras funciones discriminantes de diez colectas mexicanas de $L$. siceraria. Campo Experimental del Instituto de Ciencias Agrícolas, Universidad Autónoma de Baja California, México. 2014.

Table 4. Standarized canonical coefficients of the first two discriminant functions of ten Mexican L. siceraria landraces. Agricultural Sciences Institute Experimental field, Autonomous University of Baja California, Mexico. 2014.

\begin{tabular}{lcc}
\hline Carácter & CAN 1 & CAN 2 \\
\hline Longitud de fruto (LFR) & 0,30 & $-2,00$ \\
Circunferencia superior de fruto (CSUP) & 0,17 & 0,74 \\
Circunferencia inferior de fruto (CINF) & 1,35 & 0,50 \\
Volumen de fruto (VF) & 0,91 & 0,32 \\
Peso de 100 semillas (PCS) & 0,50 & $-0,65$ \\
Longitud de semilla (LS) & 3,84 & 13,56 \\
Diámetro aritmético de semilla (DAS) & $-4,34$ & 22,05 \\
Área estimada de semilla (AES) & $-6,79$ & $-0,35$ \\
\hline
\end{tabular}




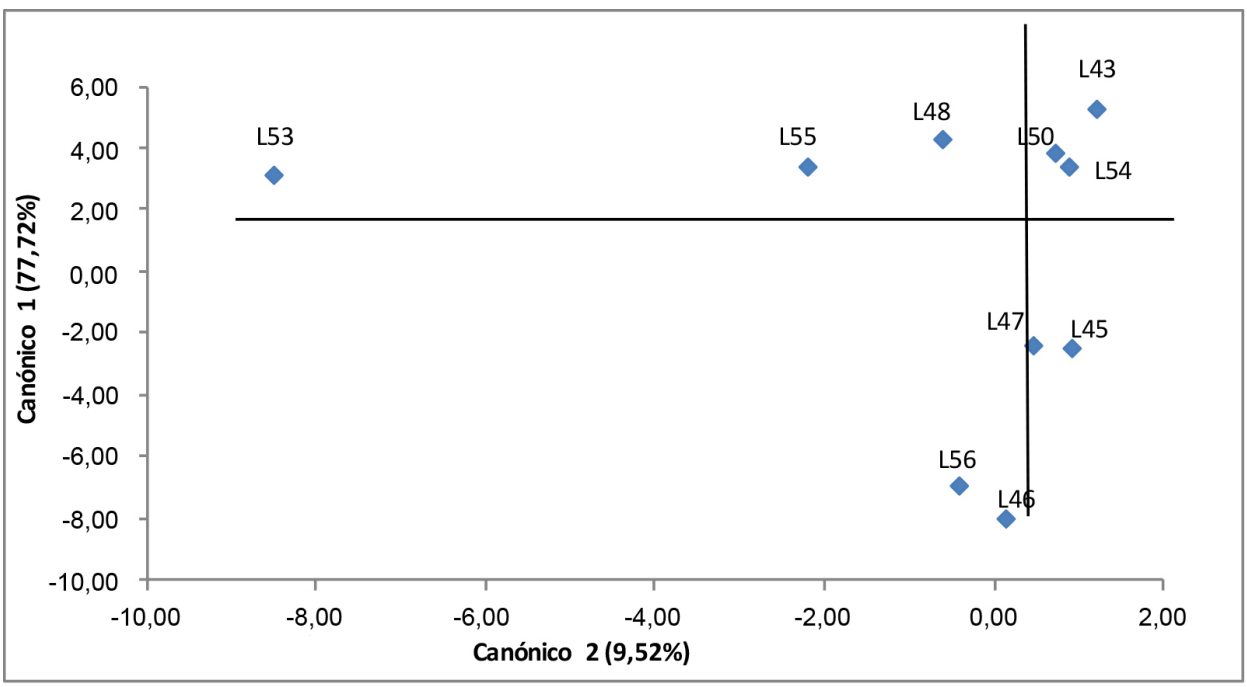

Figura 3. Distribución de diez colectas mexicanas de L. siceraria, con base en el análisis de componentes principales (ACP) de ocho caracteres seleccionados, analizados con las dos primeras funciones discriminantes canónicas. Campo Experimental del Instituto de Ciencias Agrícolas, Universidad Autónoma de Baja California, México. 2014.

Figure 3. Distribution of ten Mexican L. siceraria landraces based on the analysis of principal components (ACP) of eight selected characters, analyzed with the first two canonical discriminant function. Agricultural Sciences Institute Experimental field, Autonomous University of Baja California, Mexico. 2014.

Cuadro 5. Media y error estándar de caracteres longitud de fruto, circunferencia inferior en fruto, longitud de semillas y diámetro aritmético de semilla, en diez colectas mexicanas de L. siceraria. Campo Experimental del Instituto de Ciencias Agrícolas, Universidad Autónoma de Baja California, México. 2014.

Table 5. Mean and standard error of characters of fruit length, inferior fruit circumference, seeds length and arithmetic seed diameter of ten Mexican L. siceraria landraces. Agricultural Sciences Institute Experimental field, Autonomous University of Baja California, Mexico. 2014.

\begin{tabular}{ccccc}
\hline Colectas & $\begin{array}{c}\text { Longitud del fruto } \\
(\mathbf{c m})\end{array}$ & $\begin{array}{c}\text { Circunferencia } \\
\text { inferior }(\mathbf{c m})\end{array}$ & $\begin{array}{c}\text { Longitud de la semilla } \\
(\mathbf{c m})\end{array}$ & $\begin{array}{c}\text { Diámetro aritmético } \\
\text { de la semilla }(\mathbf{c m})\end{array}$ \\
\hline L 43 & $29,01 \pm 1,01$ & $71,63 \pm 1,88$ & $2,15 \pm 0,04$ & $1,12 \pm 0,02$ \\
L 45 & $21,70 \pm 0,43$ & $48,03 \pm 1,08$ & $1,41 \pm 0,04$ & $0,83 \pm 0,02$ \\
L 46 & $16,23 \pm 0,63$ & $30,65 \pm 0,88$ & $1,17 \pm 0,02$ & $0,70 \pm 0,01$ \\
L 47 & $23,61 \pm 0,72$ & $47,37 \pm 1,48$ & $1,48 \pm 0,02$ & $0,84 \pm 0,01$ \\
L 48 & $29,17 \pm 0,66$ & $62,65 \pm 0,90$ & $2,17 \pm 0,04$ & $1,11 \pm 0,01$ \\
L 50 & $26,79 \pm 0,86$ & $60,95 \pm 1,39$ & $2,22 \pm 0,03$ & $1,15 \pm 0,02$ \\
L 53 & $44,75 \pm 4,78$ & $68,95 \pm 7,05$ & $1,78 \pm 0,06$ & $1,00 \pm 0,04$ \\
L 54 & $27,33 \pm 0,53$ & $65,27 \pm 1,63$ & $2,07 \pm 0,06$ & $1,06 \pm 0,02$ \\
L 55 & $26,25 \pm 1,93$ & $63,63 \pm 4,72$ & $1,85 \pm 0,02$ & $1,09 \pm 0,01$ \\
L 56 & $17,87 \pm 0,25$ & $29,86 \pm 0,71$ & $1,28 \pm 0,03$ & $0,75 \pm 0,01$ \\
\hline
\end{tabular}


ejemplo la longitud de fruto fue de 21,70 a 23,61 cm, circunferencia de 47,37 a 48,03 cm, longitud de semilla de 1,41 a $1,48 \mathrm{~cm}$ y diámetro aritmético de 0,83 a $0,84 \mathrm{~cm}$. El tercer grupo lo conformaron las colectas L46 y L56, que se diferenciaron de los dos grupos anteriores porque fueron de menor dimensión. La longitud del fruto fue de 16,23 a $17,87 \mathrm{~cm}$, circunferencia de 29,86 a $30,65 \mathrm{~cm}$, longitud de semilla de 1,17 a $1,25 \mathrm{~cm}$ y diámetro aritmético de 0,70 a $0,75 \mathrm{~cm}$.

El fenograma obtenido muestra la separación de las colectas de L. siceraria en dos grupos a un nivel de disimilitud de 0,76 (Figura 4). El primer grupo se integró por las colectas L45, L46, L47 y L56, las cuales a su vez, se dividen en dos subgrupos con alto nivel de similitud, el primer subgrupo se conformó por los materiales L46 y L56, mientras que el segundo subgrupo lo integraron las dos colectas restantes. El segundo grupo se integró por las seis colectas restantes, donde L53 y L43 difirieron de las demás en niveles de disimilitud de 0,69 y 0,38, respectivamente. Las colectas L48, L50, L55 y L54 se ubicaron dentro de un mismo grupo.

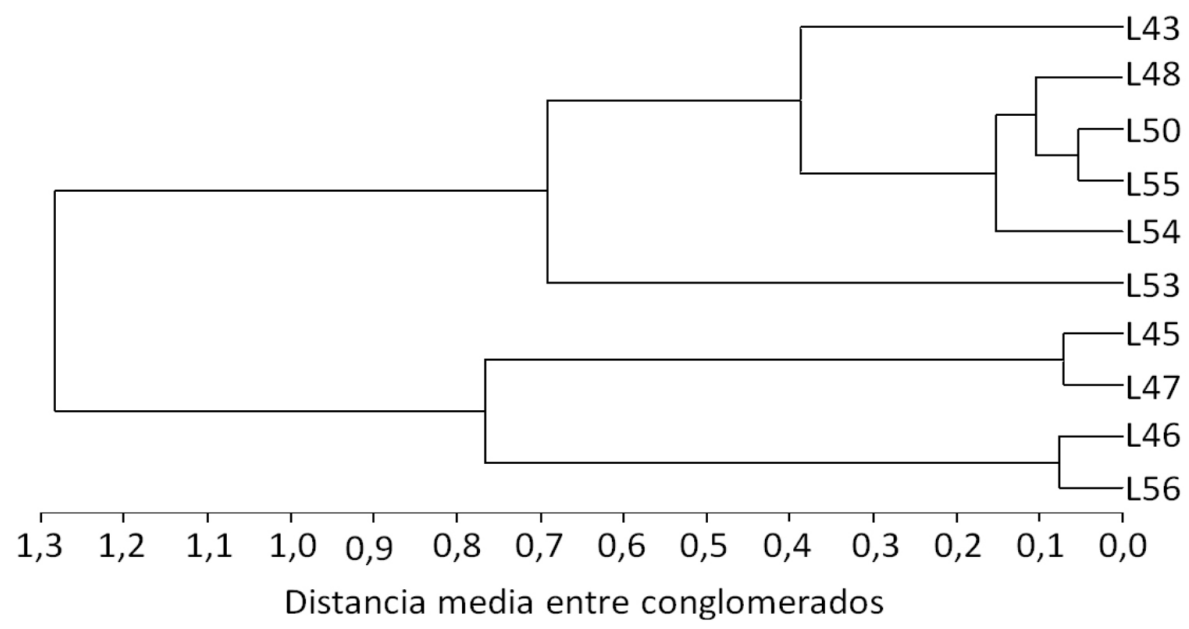

Figura 4. Fenograma de diez colectas mexicanas de L. siceraria, obtenido por la técnica de ligamiento promedio (UPGMA). Campo Experimental del Instituto de Ciencias Agrícolas, Universidad Autónoma de Baja California, México. 2014.

Figure 4. Ten Mexican L. siceraria collections Phenogram, obtained by the average linkage technique (UPGMA). Agricultural Sciences Institute Experimental field, Autonomous University of Baja California, Mexico. 2014.

\section{Discusión}

La diversidad morfológica de L. siceraria ha sido estudiada mediante la evaluación de caracteres de plántula, hoja, flor, fruto y semilla; sin embargo, se identificaron los caracteres de semilla y frutos, como los de mayor importancia en la formación de morfotipos (Yetisir et al., 2008; Koffi et al., 2009).

En semilla, la longitud, ancho, peso de 100 semillas y número de semilla por fruto, y en el fruto el volumen, han sido los más importantes descriptores en L. siceraria (Yetisir et al., 2008; Koffi et al., 2009); estos caracteres, en parte, también fueron identificados en el análisis de componentes principales en la presente investigación. En semilla, la longitud, peso de 100 semillas, diámetro aritmético y área estimada, y en fruto circunferencia superior e inferior, agruparon a las diez colectas de L. siceraria en dos grupos (Figura 2). Uno de los grupos se formó 
por las colectas L43, L48, L50, L54 y L55, las cuales compartieron mayores dimensiones en semilla y fruto con respecto al segundo grupo integrado por L45, L46, L47 y L56. Las características propias de cada grupo estuvieron relacionadas con la correlación positiva entre las dimensiones de semilla y fruto (Mladenović et al., 2012).

El fenograma generado por la similitud de caracteres entre las colectas (Figura 4), permitió identificar como principales descriptores a la longitud y diámetro aritmético de semilla, y en fruto a la longitud y circunferencia inferior. Uno de los grupos se integró por las colectas L43, L48, L50, L53, L54 y L55, que presentó las mayores dimensiones en semilla y fruto. Las semillas presentaron longitudes de 1,78 a 2,15 cm y diámetro de 1,00 a 1,12 $\mathrm{cm}$, y en el caso de longitud de fruto de 29,01 a 44,75 cm y circunferencia de 62,02 a 71,63 cm. Estas variaciones identifican a L53 y L43 como las de mayor contraste, mientras que L48, L50, L54 y L55 fueron similares. El segundo conglomerado, se distinguió del anterior por presentar semillas y frutos de menor dimensión, así como mayor similitud entre las colectas. Los subgrupos formados por L46 y L56, L45 y L47, presentaron caracteres de circunferencia inferior de fruto, longitud y diámetro aritmético de semilla altamente similares. En circunferencia inferior de fruto, las diferencias en el primer subgrupo fueron de $0,79 \mathrm{~cm}$ y en el segundo de $0,66 \mathrm{~cm}$. En cuanto a longitud y diámetro aritmético de semilla, existió mayor uniformidad, ya que, las diferencias entre los materiales no superó los $11 \mathrm{~mm}$ en ambos subgrupos. Estas diferencias entre los materiales difícilmente podrían ser reconocidas para identificar fenotipos diferentes y genéticamente serían declarados similares. Sin embargo, al considerar el lugar de procedencia de L46 que es el estado de Oaxaca y L56 del estado de Morelos, se tiene la posibilidad de que genéticamente sean diferentes a pesar de compartir similitud en los caracteres. Para esclarecer esta condición es conveniente en estudios posteriores integrar determinaciones con técnicas moleculares. En la condición de los materiales L45 y L47, puesto que ambos comparten valores similares en los caracteres y que provienen de la región de Mexicali, Baja California, se tiene mayor certeza de que genéticamente son similares, aunque al igual que en el caso anterior, es necesario integrar otras variables de estudio para diagnosticar con mayor precisión las relaciones genéticas entre los materiales.

La integración de las diez colectas en morfotipos diferentes, mostró que hay variabilidad genética de la especie de L. siceraria en los materiales nativos de México; esta condición, genera la posibilidad de evaluar y seleccionar materiales de Lagenaria con potencial de uso como portainjerto. Además, de establecer la relación entre los caracteres morfológicos y la tolerancia a patógenos y respuesta productiva en cucurbitáceas en la modalidad injertada.

\section{Conclusiones}

El presente estudio evidenció que Lagenaria siceraria en México fue variable morfológicamente en características de longitud y diámetro de semilla, y en fruto en su longitud y circunferencia inferior. El reconocimiento de diferentes morfotipos demuestra variabilidad genética de las materiales nativos de México y genera la posibilidad de seleccionar materiales con mayor potencial de uso como portainjertos para la producción de cucurbitáceas.

\section{Agradecimientos}

A la Universidad Autónoma de Baja California, por el apoyo otorgado a través de la $17^{\text {a }}$ Convocatoria Interna de Proyectos de Investigación. 


\section{Literatura citada}

Bevacqua, R.F. 1994. Origin of horticulture in Southeast Asia and the dispersal of domesticated plants to the Pacific Islands by Polynesian voyagers: the Hawaiian Islands case study. HortSci. 29:1226-1229.

Chimonyo, V.G.P., and A.T. Modi. 2013. Seed performance of selected bottle gourd (Lagenaria siceraria (Molina) Standl.). Am. J. Exp. Agric. 3:740-766. doi:10.9734/ajea/2013/4114

Decker-Walters, D., J. Staub, A. López-Sesé, and E. Nakata. 2001. Diversity in landraces and cultivars of bottle gourd (Lagenaria siceraria; Cucurbitaceae) as assessed by random amplified polymorphic DNA. Gen. Resour. Crop Evol. 48:369-380. doi:10.1023/A:1012079323399

Erickson, D.L., B.D. Smith, A.C. Clarke, D.H. Sandweiss, and N. Tuross. 2005. An Asian origin for a 10,000-year-old domesticated plant in the Americas. Proc. Natl. Acad. Sci. 102:18315-18320. doi:10.1073/pnas.0509279102

Gürcan, K., A. Say, H. Yetisir, and N. Denli. 2015. A study of genetic diversity in bottle gourd [Lagenaria siceraria (Molina) Standl.] population, and implication for the historical origins on bottle gourds in Turkey. Gen. Resour. Crop Evol. 62:321333. doi:10.1007/s10722-015-0224-8

Guzmán-Hernández, E.A., D. Segura-Cobos, and P. López-Sánchez. 2016. Plants present in Mexico with studies in metabolic syndrome. J. Med. Plants Stud. 4:95-103.

Karaca, F., H. Yetisir, I. Solmaz, E.E. or-Çandir, S. Kurt, N. Sari, and Z. Guler. 2012. Rootstock potential of Turkish Lagenaria siceraria germplasm for watermelon: plant growth, yield and quality. Turk. J. Agric. For. 36:167-177. doi:10.3906/tar1101-1716

Kelley, W.M., D.M. Granberry, and G.E. Boyhan. 2009. Soils and fertility. In: W.T. Kelley, and D.B. Lagston, editors, Commercial production and management of pumpkins and gourds. University of Georgia Cooperative Extension, USA. p. 11-12.

Kistler, L., A. Montenegro, B.D. Smith, J.A. Gifford, R.E. Green, L.A. Newsom, and B. Shapiro. 2014. Transoceanic drift and the domestication of African bottle gourds in the Americas. Proc. Natl. Acad. Sci. 111:2937-2941. doi:10.1073/ pnas. 1318678111

Koffi, K.K., G.K. Anzara, M. Malice, Y. Djè, P. Bertin, J.P. Baudoin, and I.A. Zoro-Bi. 2009. Morphological and allozyme variation in a collection of Lagenaria siceraria (Molina) Standl. from Côte d'Ivoire. Biotechnol. Agron. Soci. Environ. 13:257-270.

Kubde, M.S., S.S. Khadabadi, I.A. Farooqui, and S.L. Deore. 2010. Lagenaria siceraria: phytochemistry, pharmacognosy and pharmacological studies. Rep. Opin. 2:91-98.

López-Elías, J., M.A. Huez-López, J. Jimenez-León, J.C. Rodríguez, S. Garza-Ortega, y L.F. Escoboza-García. 2011. Efecto de la densidad de plantación en sandia sin semilla injertada sobre bule (Lagenaria siceraria (Molina) Standl). Trop. Subtrop. Agroecosyst. 14:349-355.

Miguel, A., J.V. Maroto, A. San-Bautista, C. Baixauli, V. Cebolla, B Pascual, S. López, and J.L. Guardiola. 2004. The grafting of triploid watermelon is an advantageous alternative to soil fumigation. Sci. Hort. 103:9-17. doi:10.1016/j. scienta.2004.04.007

Mladenović, E., J. Berenji, V. Ognjanov, M. Ljubojević, and J. Čukanović. 2012. Genetic variability of bottle gourd Lagenaria siceraria (mol.) Standley and its morphological characterization by multivariate analysis. Arch. Biol. Sci. 64:573-583. doi:10.2298/ABS1202573M 
Morimoto, Y., P. Maundu, H. Fujimaki, and H. Morishima. 2005. Diversity of landraces of the white-flowered gourd (Lagenaria siceraria) and its wild relatives in Kenya: fruit and seed morphology. Gen. Resour. Crop Evol. 52:737-747. doi:10.1007/ s10722-004-6119-8

Pradhan, R.C., P.P. Said, and S. Singh. 2013. Physical properties of bottle gourd seeds. Agric. Eng. Int.: CIGR J. 15(1):106-113. SAS, 2009. SASTSATAT 9.2. User's guide. $2^{\text {nd }}$ ed. SAS Institute Inc., Cary, NC, USA.

Smith, B.D. 2005. Reassessing Coxcatlan Cave and the early history of domesticated plants in Mesoamerica. Proc. Natl. Acad. Sci. USA. Proc. Natl. Acad. Sci. 102:9438-9445. doi:10.1073/pnas.0502847102

Suárez-Hernández, A.M., O. Grimaldo-Juárez, A.M. García-López, D. González-Mendoza, y M.V. Huitron-Ramírez. 2017. Influencia del portainjerto en la calidad poscosecha de sandia. Rev. Chapingo Serie Hort. 23:49-58. doi:10.5154/r. rchsc. 2016.06 .019

Teppner, H. 2004. Notes on Lagenaria and Cucurbita (Cucurbitaceae) - Review and new contributions. Phyton (Horn.) 44:245308.

Yetisir, H., S. Kurt, N. Sari, and F.M. Tok. 2007. Rootstock potential of turkish Lagenaria siceraria germplasm for watermelon: plant growth, graft compatibility, and resistance to Fusarium. Turk. J. Agric. For. 31:381-388.

Yetisir, H., M. Sakar, and S. Serçe. 2008. Collection and morphological characterization of Lagenaria siceraria germplasm from the Mediterranean region of Turkey. Gen. Resour. Crop Evol. 55:1257-1266. doi:10.1007/s 10722-008-9325-y

Yetisir, H., and N. Sari. 2003. Effect of different rootstock on plant growth, yield and quality of watermelon. Aust. J. Exp Agr. 43:1269-1274.

Yetisir, H., and N. Sari. 2003. Effect of different rootstock on plant growth, yield and quality of watermelon. Aust. J. Exp Agr. 43:1269-1274.

Yetisir, H., F. Kurt, N. Sari, and F.M. Tok. 2007. Rootstock potential of turkish Lagenaria siceraria germplasm for watermelon: plant growth, graft compatibility, and resistance to Fusarium. Turk. J. Agric. For. 31:381-388.

Yetisir, H., M. Sakar, and S. Serce. 2008. Collection and morphological characterization of Lagenaria siceraria germplasm from the Mediterranean region of Turkey. Gen. Resour. Crop Evol. 55:1257-1266. 